\title{
$\mathrm{Li}_{10} \mathrm{GeP}_{2} \mathrm{~S}_{12}$ 固态电解质电极界面特性研究
}

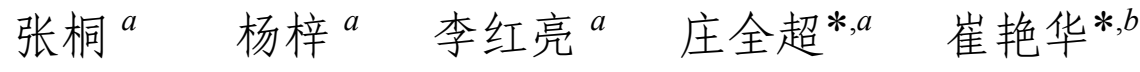 \\ ( ${ }^{a}$ 中国矿业大学 材料科学与工程学院 徐州 221116) \\ $\left({ }^{b}\right.$ 中国工程物理研究院 电子工程研究所 绵阳 621900)
}

\begin{abstract}
摘要 采用高温固相法合成了固态电解质 $\mathrm{Li}_{10} \mathrm{GeP}_{2} \mathrm{~S}_{12}$, 其室温离子电导率为 $2.02 \times 10^{-3} \mathrm{~S} / \mathrm{cm}$, 并组装了 $\mathrm{LiNbO}_{3} @ \mathrm{LiNi}_{1 / 3} \mathrm{Co}_{1 / 3} \mathrm{Mn}_{1 / 3} \mathrm{O}_{2} / \mathrm{Li}_{10} \mathrm{GeP}_{2} \mathrm{~S}_{12} / \mathrm{Li}$ 全固态电池. 恒流充放电测试表明全固态电池首次放电容量 $121.2 \mathrm{mAh} / \mathrm{g}$, 库伦效率 40 周后稳定在 $99.8 \%$ 左右, 循环 100 周后容量保持率达 $93.7 \%$. 电化学阻抗谱的测试结果表明, 其典型的阻抗 谱图由高频区半圆(HFS)、中频区半圆(MFS)和低频区斜线( $\mathrm{LFL}$ )组成, 其中, HFS 归属于电解质阻抗 $\left(R_{\mathrm{el}} / / Q_{\mathrm{el}}\right), \mathrm{MFS}$ 归属 于电荷传递过程 $\left(R_{\mathrm{cc}} / / Q_{\mathrm{dl}}\right), \mathrm{LFL}$ 归属于锂离子的固态扩散过程. 通过选取适当的等效电路, 对实验所得的电化学阻抗谱 数据进行拟合, 并分析了 $R_{\mathrm{el}}$ 和 $R_{\mathrm{ct}}$ 随电极电位的变化规律.

关键词 全固态电池; $\mathrm{Li}_{10} \mathrm{GeP}_{2} \mathrm{~S}_{12}$; 电化学阻抗谱; 电荷传递电阻
\end{abstract}

\section{Electrochemical Impedance Spectroscopic Studies of All Solid State battery with $\mathrm{Li}_{10} \mathrm{GeP}_{2} \mathrm{~S}_{12}$ as Electrolyte}

\author{
Zhang, Tong $^{a} \quad{\text { Yang, } \mathrm{Zi}^{a} \quad \mathrm{Li} \text {, Hongliang }}^{a} \quad$ Zhuang, Quanchao*,a $\quad$ Cui, Yanhua*, \\ $\left({ }^{a}\right.$ School of Materials Science and Engineering, China University of Mining and Technology, Xuzhou 221116) \\ ( ${ }^{b}$ Institute of Electronic Engineering, China Academy of Engineering Physics, Mianyang 621900)
}

\begin{abstract}
All-solid-state batteries will be the main direction of lithium-ion batteries in the future. Current research mainly focuses on improving the conductivity of solid-state electrolytes, but there are few studies on the electronic and ionic transport in all solid state batteries. In this paper, we synthesized $\mathrm{Li}_{10} \mathrm{GeP}_{2} \mathrm{~S}_{12}$ through high temperature solid phase method. The ionic conductivity of $\mathrm{Li}_{10} \mathrm{GeP}_{2} \mathrm{~S}_{12}$ at room temperature is $2.02 \times 10^{-3} \mathrm{~S} / \mathrm{cm}$ and it's activation energy calculated from Arrhenius plots is $29.8 \mathrm{~kJ} / \mathrm{mol}$. The all solid-state battery of $\mathrm{LiNbO}_{3} @ \mathrm{LiNi}_{1 / 3} \mathrm{Co}_{1 / 3} \mathrm{Mn}_{1 / 3} \mathrm{O}_{2} / \mathrm{Li}_{10} \mathrm{GeP}_{2} \mathrm{~S}_{12} / \mathrm{Li}$ was successfully fabricated and characterized by galvanostatic charge/discharge (DC), cyclic voltammetry (CV) and electrochemical impedance spectroscopy (EIS). The first discharge capacity of the all-solid-state battery is $121.2 \mathrm{mAh} / \mathrm{g}$, the coulombic efficiency stabilize at $99.8 \%$ after 40 weeks and the capacity retention rate is $93.7 \%$ after 100 weeks. After analyzing the electrochemical impedance spectroscopy, the typical impedance spectra of the battery is composed of high frequency semicircle (HFS), middle frequency semicircle (MFS) and low frequency line (LFL). And HFS belongs to the impedance of electrolyte $\left(R_{\mathrm{el}}\right)$, MFS belongs to charge transfer impedance $\left(R_{\mathrm{ct}}\right)$ and LFL belongs to diffusion process of lithium ion in active material. The continuous increase of $R_{\mathrm{el}}$ between $3.8 \mathrm{~V}$ and $4.3 \mathrm{~V}$ is due to the decomposition of LGPS to $\mathrm{GeS}_{2}, \mathrm{~S}_{\text {and }} \mathrm{P}_{2} \mathrm{~S}_{5}$ at high potential, which results in the decrease of grain conductivity. On the other hand, the voltage range of $3.8 \sim 4.3 \mathrm{~V}$ is near the charging and discharging plateau at which concentration polarization is large. The stress in the crystal may lead to the breakup of some grains which resulting in the generation of more grain boundaries and the increase of grain boundary impedance. According to the fitting results of $R_{\mathrm{ct}}$, we find that $R_{\mathrm{ct}}$ decreases with the increase of potential until $4.3 \mathrm{~V}$ at which $R_{\mathrm{ct}}$ reaches the minimum value in the first process of charging and it is a reversible process while discharging.

Keywords all solid state batteries; $\mathrm{Li}_{10} \mathrm{GeP}_{2} \mathrm{~S}_{12}$; electrochemical impedance spectroscopy; charge transfer resistance
\end{abstract}

\section{1 引言}

全固态电池是未来锂电发展的大方向，有望在实现 高能量密度、长循环寿命的同时从根本上解决锂离子电 池的安全性问题, 是未来电动汽车和规模化储能理想的 化学电源 ${ }^{[17]}$.

目前, 关于全固态电池的研究主要集中在提高固态 电解质的离子电导率 ${ }^{[8 \sim 15]}$ 以及如何改善电极/电解质界
面性质，增进电极/电解质界面兼容性 ${ }^{[16 ~ 29]}$, 特别是正 极/电解质界面的兼容性上. 硫化物被认为是锂离子电 池全固态化的最佳电解质材料之一, 由于硫化物固态电 解质仅仅通过冷压就能明显降低晶界电阻, 因此具有良 好的室温离子电导率，其室温离子电导率可以达到能与 液体电解质相媲美的程度 ${ }^{[30,31]}$. 电化学阻抗谱(EIS)技 术对表征电化学反应过程具有独特的优势, 它能够根据

\footnotetext{
* E-mail: zhuangquanchao@126.com; cuiyanhua@netease.com

Received January 5, 2019; published May 9, 2019.

Project supported by the National Natural Science Foundation of China (No. U1730136).

项目受国家自然科学基金(No. U1730136)资助.
} 
电化学反应过程中每一步骤弛豫时间常数的不同, 在较 宽频率范围对其表征. 通过等效电路对 EIS 谱数据进行 拟合分析, 可以获得锂离子在全固态锂电池中与输运过 程相关的电极过程动力学参数, 为优化电解质设计和界 面控制技术提供理论指导, 然而目前运用 EIS 研究电极/ 全固态电解质界面性质相对较少 ${ }^{[32 ~ 34]}$. 因此, 在本研究 中, 我们以 $\mathrm{LiNbO}_{3} @ \mathrm{LiNi}_{1 / 3} \mathrm{Co}_{1 / 3} \mathrm{Mn}_{1 / 3} \mathrm{O}_{2}$ 为正极(简称 LNCM111, $\mathrm{LiNi}_{1 / 3} \mathrm{Co}_{1 / 3} \mathrm{Mn}_{1 / 3} \mathrm{O}_{2}$ 简称 $\mathrm{NCM} 111$ ), 以 $\mathrm{Li}_{10^{-}}$ $\mathrm{GeP}_{2} \mathrm{~S}_{12}$ 为电解质(简称 LGPS), 锂金属为负极组装了全 固态电池, 运用 $\mathrm{EIS}$ 研究了 $\mathrm{LiNbO}_{3} @ \mathrm{LiNi}_{1 / 3} \mathrm{Co}_{1 / 3}$ $\mathrm{Mn}_{1 / 3} \mathrm{O}_{2} / \mathrm{Li}_{10} \mathrm{GeP}_{2} \mathrm{~S}_{12} / \mathrm{Li}$ 全固态电池(简称 LGPS 全固态电 池)的首次充放电过程.

\section{2 结果与讨论}

\section{1 材料的物相和形貌分析}

图 1 为 LGPS 的 XRD 测试结果, 可以看到, 合成材 料的 XRD 峰的位置和相对峰强度均与计算结果相符, 表明成功合成了该材料.

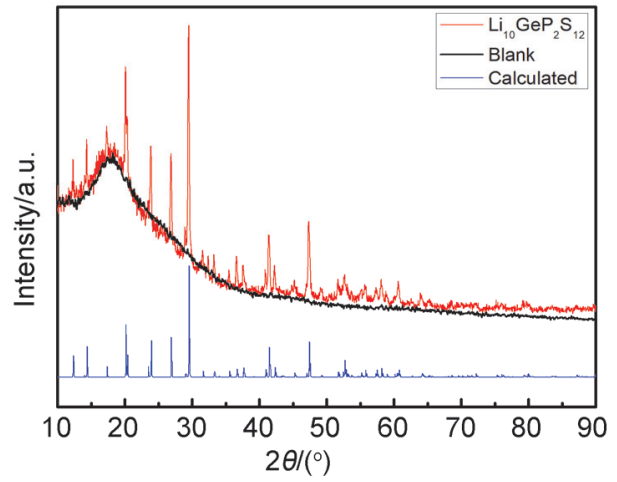

图 1 LGPS 的 XRD 衍射图

Figure 1 X-ray diffraction patterns of LGPS

图 2 为 LNCM111 和 NCM111 的 XRD 测试结果, 由 图可知, 包覆前后材料的 XRD 谱峰基本没有发生变化 且没有与 $\mathrm{LiNbO}_{3}$ 相关谱峰出现, 表明包覆过程没有对 NCM111 的结构产生影响, 包覆层较薄. 为了进一步说 明在 NCM111 表面成功进行了包覆, 我们测试了

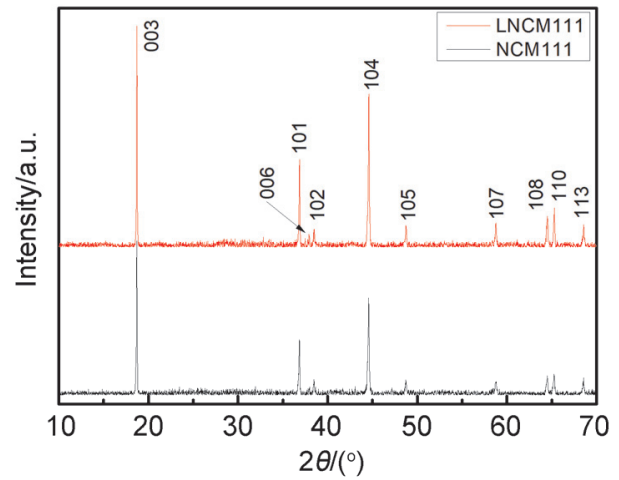

图 2 LNCM111 和 NCM111 的 XRD 衍射图

Figure 2 X-ray diffraction patterns of LNCM111 and NCM111
LNCM111 和 NCM111 的 SEM 图，如图 3. 可以看出，包 覆前的 NCM111 是由粒径约 $200 \mathrm{~nm} \sim 1 \mu \mathrm{m}$ 的小颗粒团 聚而成的粒径约 3 4 $\mu \mathrm{m}$ 大颗粒, 表面光滑, 而包覆后 的 LNCM111 表面变得粗糙, 原本小颗粒清晰的轮廓已 经变得模糊, 表明我们成功的在 NCM111 表面包覆了一 层 $\mathrm{LiNbO}_{3}$. 图 4 为 LNCM111 的 Mapping 测试结果, 可 以直观地看到 $\mathrm{Nb}$ 元素均匀地分布在整个活性物质颗粒 上, 说明表面包覆比较均匀.

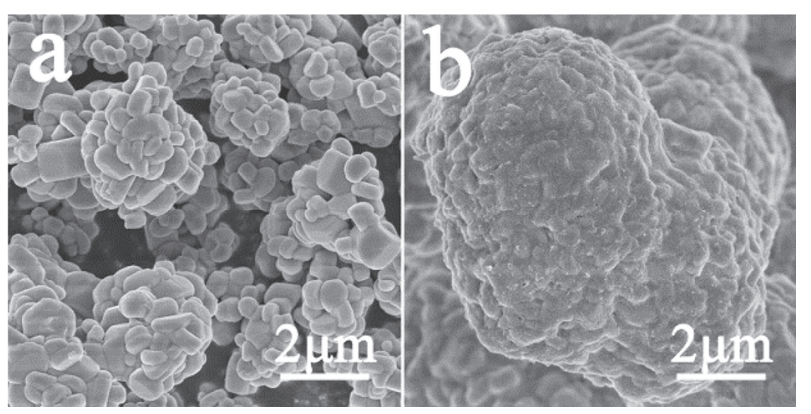

图 3 (a) NCM111 和(b) LNCM111 的 SEM 图

Figure 3 SEM images of (a) NCM111 and (b) LNCM111

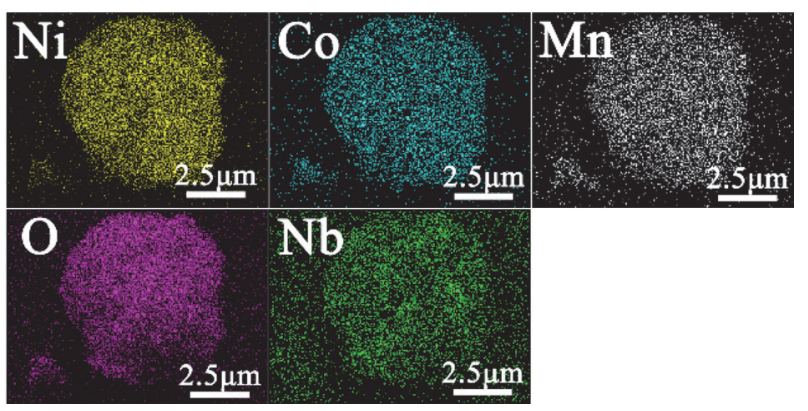

图 4 LNCM111 的 Mapping 图

Figure 4 Mapping images of LNCM111

\section{2 电化学性能测试分析}

图 5a 是用全阻塞电池 $\mathrm{SS} / \mathrm{LGPS} / \mathrm{SS}$ (SS 表示不锈钢 片)在不同温度下测试的交流阻抗图，一般由一个半圆 和一条斜线组成. 一般认为 ${ }^{[35]}$, 斜线与实轴的交点可近 似作为电解质的晶粒电阻 $R_{\mathrm{b}}$ 和晶界电阻 $R_{\mathrm{gb}}$ 之和, 可以 用于计算离子电导率.

图 5b 为从图 5a 计算得到的 LGPS 的 Arrhenius 曲 线, 可以看出, $\ln \sigma$ 与 $1000 / T$ 呈现较好的线性关系, 其中 LGPS 室温下的离子电导率为 $2.02 \times 10^{-3} \mathrm{~S} / \mathrm{cm}$. 进一步 从 LGPS 的 Arrhenius 曲线计算获得其活化能为 29.8 $\mathrm{kJ} / \mathrm{mol}$. 图 $5 \mathrm{c}$ 为 $\mathrm{SS} / \mathrm{LGPS} / \mathrm{Li}$ 在 $0 \sim 6 \mathrm{~V}$ 的 CV 测试结果, 可以看到 LGPS 在 $0 \sim 6 \mathrm{~V}$ 对锂稳定性较好, 与之前的文 献相符 ${ }^{[31,36]}$.

图 6 为 LGPS 全固态电池的充放电曲线、循环性能 曲线和 $\mathrm{CV}$ 曲线. 可以看出, 其首周放电容量为 121.2 $\mathrm{mAh} / \mathrm{g}$ ，库伦效率 $58.7 \%$ ，第二周库伦效率上升到 $93.9 \%$, 并在 40 周以后稳定在 $99.8 \%$ 左右, 经过 100 周 


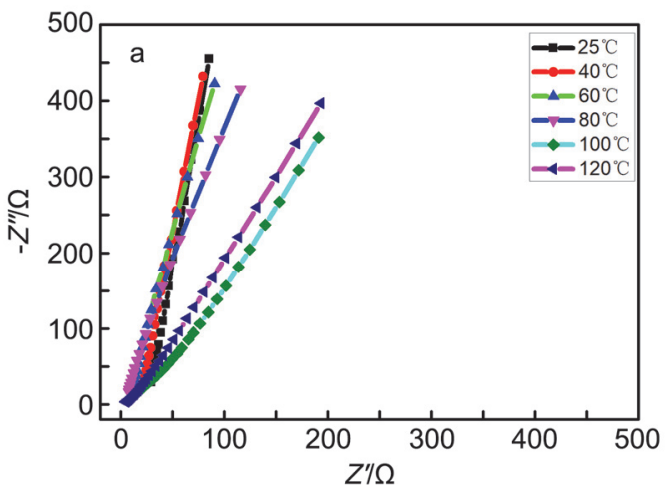

Temperature $/{ }^{\circ} \mathrm{C}$
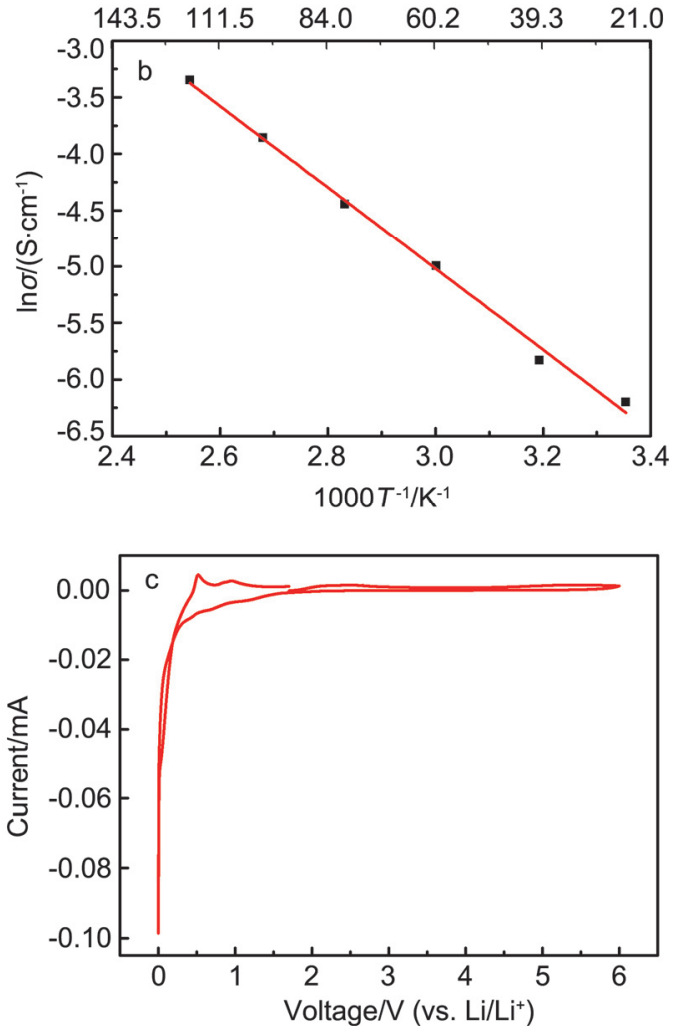

图 5 (a) SS/LGPS/SS 不同温度下的交流阻抗谱图. (b) LGPS 的 Arrhenius 曲线. (c) SS/LGPS/Li 的 CV 曲线

Figure 5 (a) Electrochemical impedance spectroscopy measured with SS/LGPS/SS cell at different temperatures. (b) Arrhenius conductivity plots of LGPS. (c) Cyclic voltammetry curve of SS/LGPS/Li cell

的循环, 其放电容量为 $113.6 \mathrm{mAh} / \mathrm{g}$, 容量保持率 $93.7 \%$, 显示了优异的电化学性能. 从图 $6 c$ 可以看出, 其氧化峰在 $3.9 \mathrm{~V}$ 左右, 还原峰在 $3.73 \mathrm{~V}$ 左右, 与图 $6 \mathrm{a}$ 中的充放电平台相对应, 和 NCM111 作为正极活性材料 的液态电池相比 ${ }^{[37]}$, 极化稍偏大, 这应该是其放电容量 偏低的原因. 此外, 第二周以后的 $\mathrm{CV}$ 曲线几乎重合, 说明电池的循环性能和可逆性很好, 与充放电结果相符 合.

\subsection{EIS 测试分析}

图 7 和图 8 为 LGPS 全固态电池首次充放电过程中
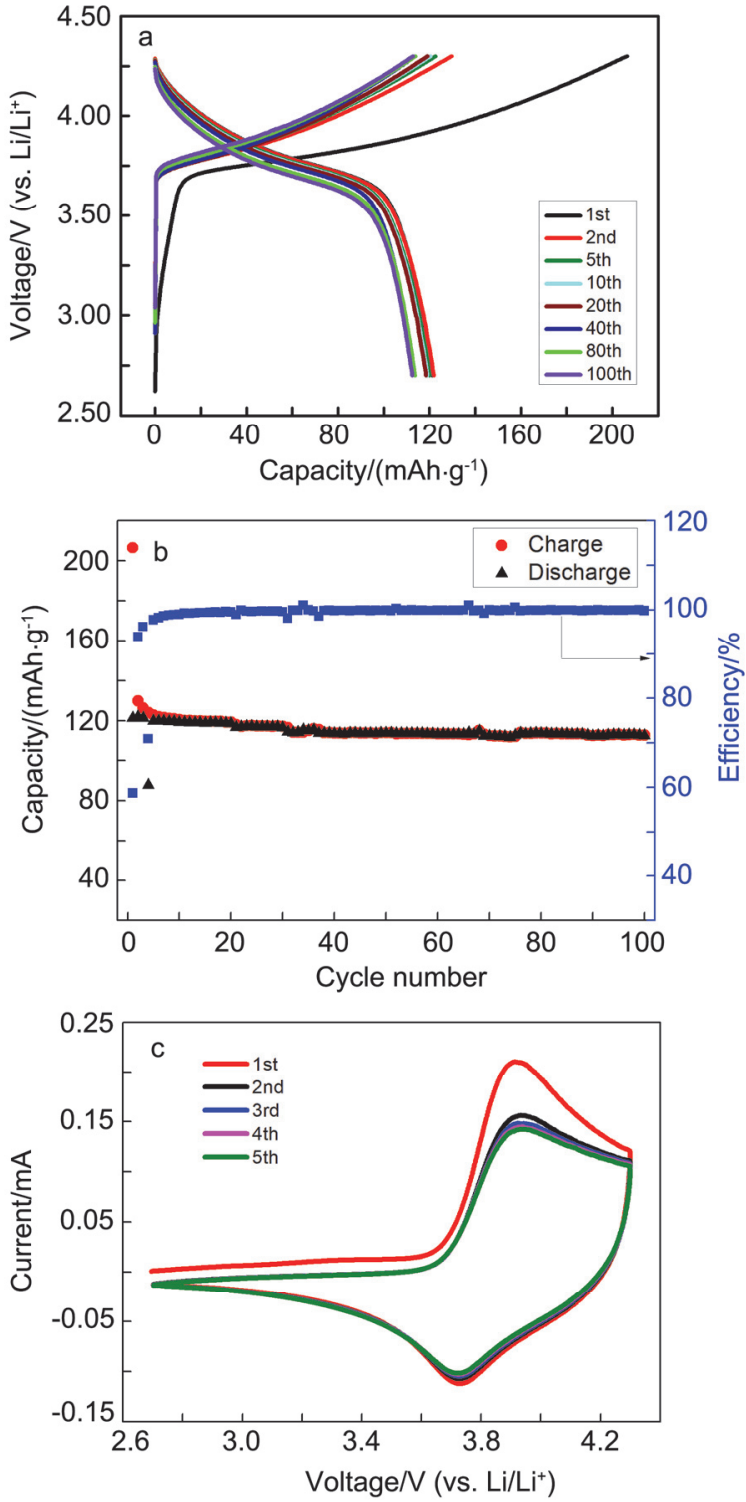

图 6 LGPS 全固态电池的(a)充放电曲线, (b)循环性能曲线和(c) CV 曲线

Figure 6 (a) Charge/discharge curves, (b) Cycle performance curves and (c) Cyclic voltammetry curves of the all solid state battery with LGPS as electrolyte

2.7 4.3 V 下的 EIS 谱图. 可以看出, LGPS 全固态电池 在开路电位 $2.7 \mathrm{~V}$ 下, Nyquist 图由高频区域一个较小的 半圆(HFS)和中低频区域的斜线组成. 随着电位的升高, 在 2.8 3.4 V, HFS 基本保持不变; 但中低频区域的斜线 对电位显示出明显的依赖, 不断向实轴弯曲, 至 $3.5 \mathrm{~V}$, 低频区的斜线演变成为中频区域的一个半圆(MFS)和低 频区域的一条斜线(LFL). 此时, EIS 谱由三个部分组成, 即高频区半圆(HFS)、中频区半圆(MFS)和低频区斜线 (LFL). 随着电位进一步升高至 $4.3 \mathrm{~V}, \mathrm{LGPS}$ 全固态电池 的阻抗谱特征没有再发生变化. 在随后的首次放电过程 中, EIS 谱特征变化规律与其在首次充放电过程中的变 化规律表现为一个可逆的过程. 

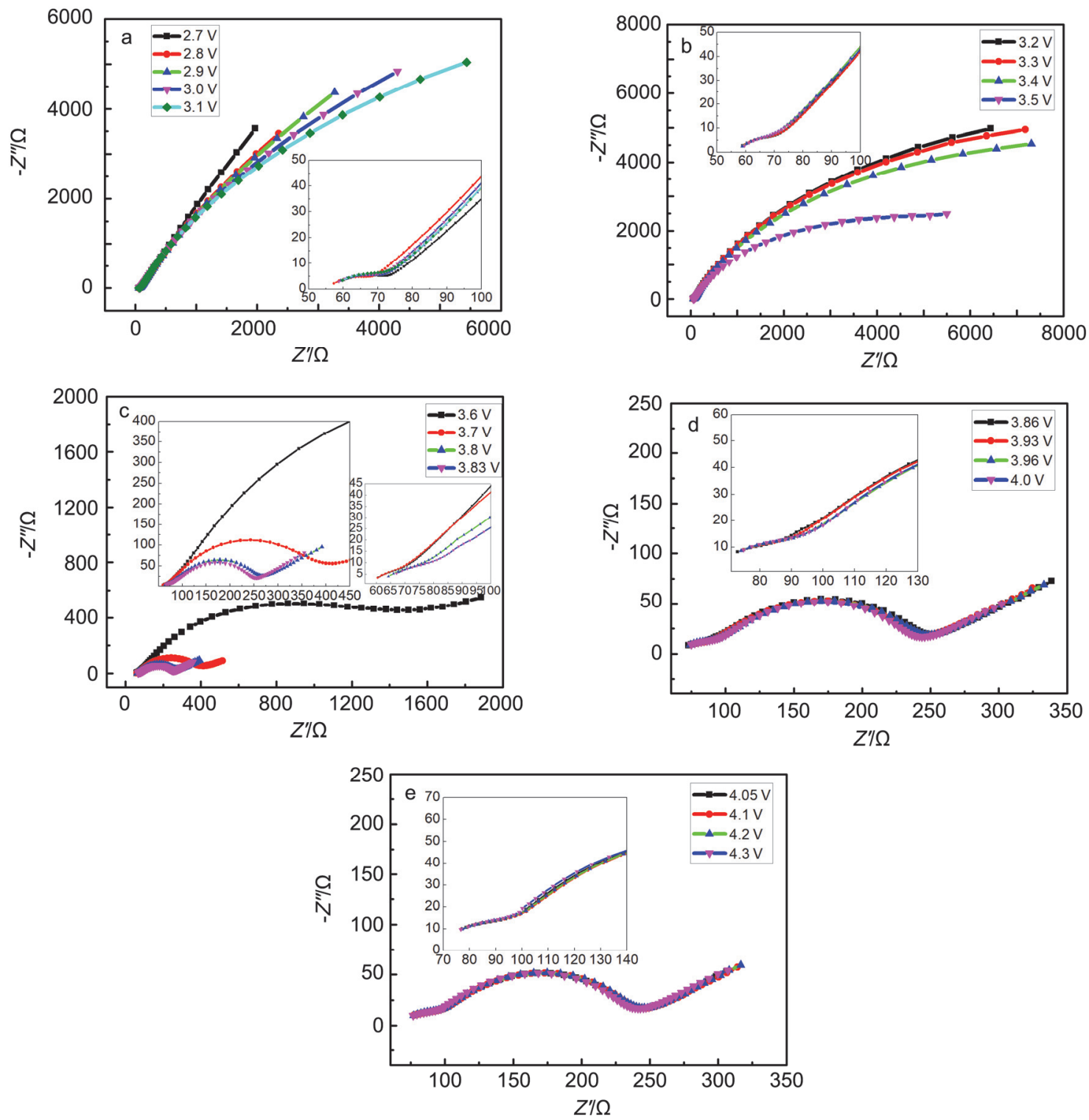

图 7 LGPS 全固态电池首次充电过程 $2.7 \sim 4.3 \mathrm{~V}$ 下的 Nyquist 图

Figure 7 Nyquist plots of the all solid state battery with LGPS as electrolyte at a series of potentials from 2.7 to $4.3 \mathrm{~V}$ during the first charging process

固态电解质一般为多晶材料, 因此其阻抗主要来自 于晶粒阻抗和晶界阻抗. 电池工作时, 离子由电极分别 经电解质晶粒和晶界, 电极/电解质界面到达另一电极. 其电池示意图如图 9 所示, 其中 $R_{\mathrm{b}}$ 和 $C_{\mathrm{b}}$ 分别表示晶粒 电阻和晶粒几何电容, $R_{\mathrm{gb}}$ 和 $C_{\mathrm{gb}}$ 分别表示晶界电阻和晶 界电容, $R_{\mathrm{ct}}$ 和 $C_{\mathrm{dl}}$ 表示电荷传递电阻与双电层电容 ${ }^{[38]}$. 相比于晶粒阻抗, 晶界阻抗往往占据主导地位, 也就是 说晶界效应通常是导致非常低的总离子电导率的关键 因素. 然而对硫化物固态电解质而言, 即使在冷压条件 下, 其晶界电阻也非常小, 因此硫化物固态电解质电池 的高频区域往往只出现与晶粒阻抗有关的一个半圆或 二者融合且以晶粒阻抗为主的一个半圆 ${ }^{[30,31]}$, 此时, 硫 化物固态电解质全固态电池的典型 EIS 谱特征由三部分
组成，即高频区半圆(HFS)、中频区半圆(MFS)和低频区 斜线(LFL).

因此, 我们将 Nyquist 图中的 HFS 归属于以晶粒阻 抗为主的电解质阻抗, MFS 归属于电荷传递过程, LFL 归属于锂离子在材料中的固态扩散过程.

根据以上分析, 我们给出了如图 10 所示的等效电 路对实验所获得的 Nyquist 图进行拟合. 其中, $R_{\mathrm{S}}$ 代表欧 姆电阻, $R_{\mathrm{el}}$ 代表电解质电阻 (以 $R_{\mathrm{b}}$ 为主), $R_{\mathrm{ct}}$ 为电荷传递 电阻, 恒相角元件(CPE) $Q_{\mathrm{el}} 、 Q_{\mathrm{dl}}$ 和 $Q_{\mathrm{D}}$ 分别代表电解质 电容 $Q_{\mathrm{el}}$ ，双电层电容 $Q_{\mathrm{dl}}$ 以及扩散阻抗. 图 11 为首次充 电过程中 $4.0 \mathrm{~V}$ 的拟合图, 从图中可以看出, 拟合曲线 和实验曲线重合性较好, 且其拟合误差 $\chi^{2}$ 值在 0.001 左 右. 

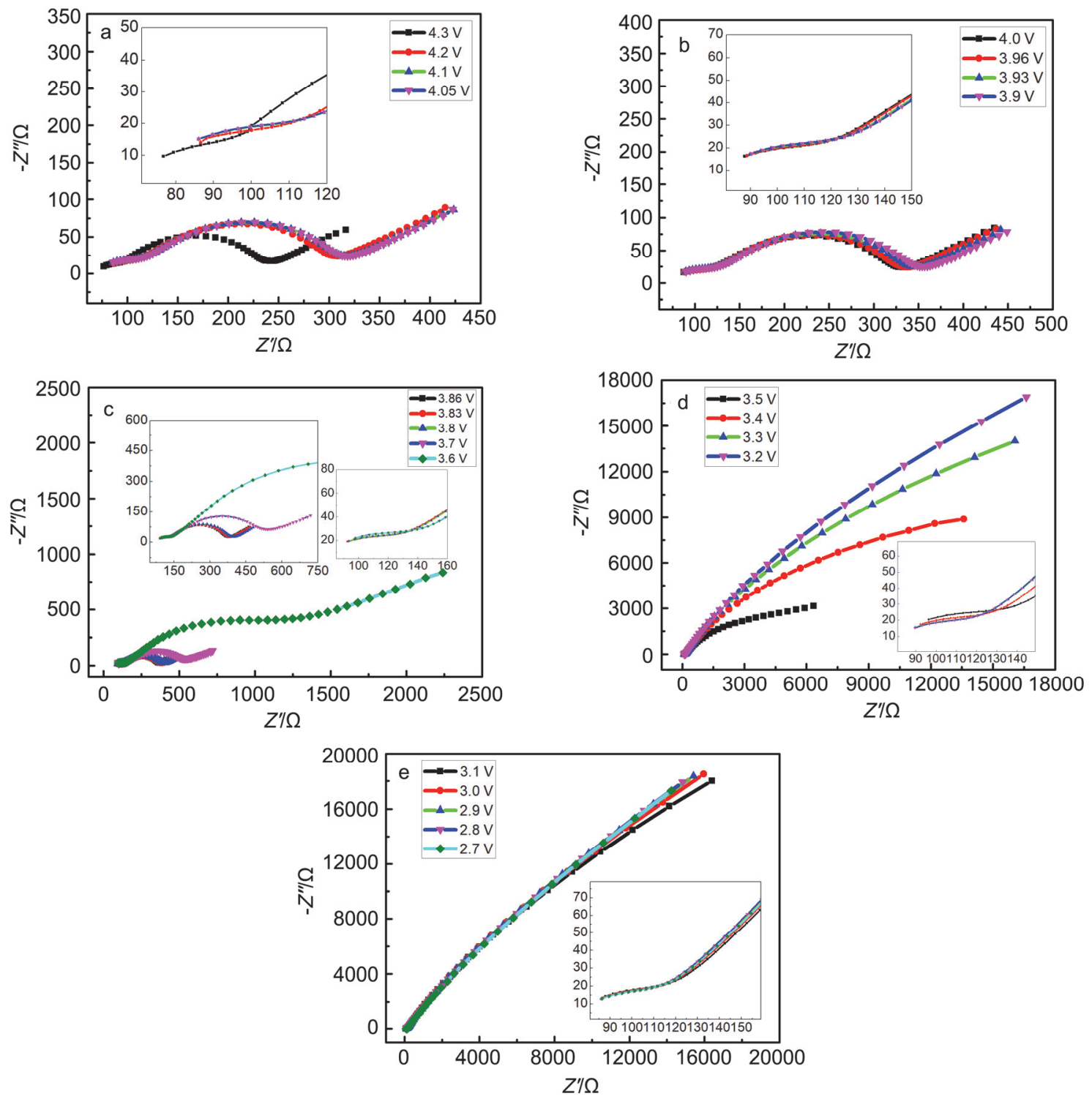

图 8 LGPS 全固态电池首次放电过程 4.3 2.7 V 下的 Nyquist 图

Figure 8 Nyquist plots of the all solid state battery with LGPS as electrolyte at a series of potentials from 4.3 to $2.7 \mathrm{~V}$ during the first discharging process

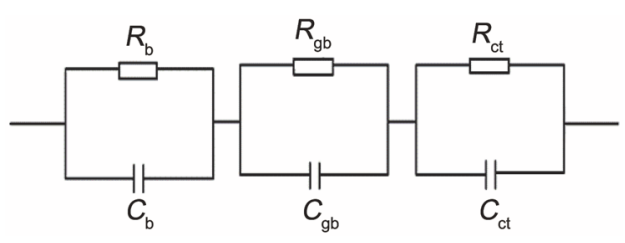

图 9 全固态电池的等效电路

Figure 9 Equivalent circuit of all solid state battery

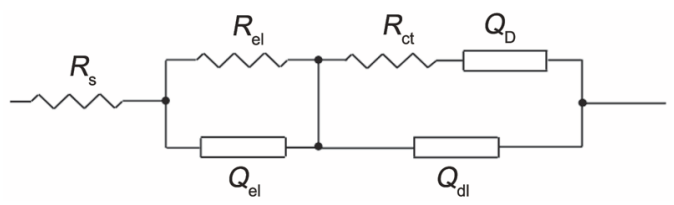

图 10 LGPS 全固态电池首次充放电 $\mathrm{EIS}$ 等效电路

Figure 10 Equivalent circuit for the analysis of the first charging/discharging process of the LGPS all solid state battery

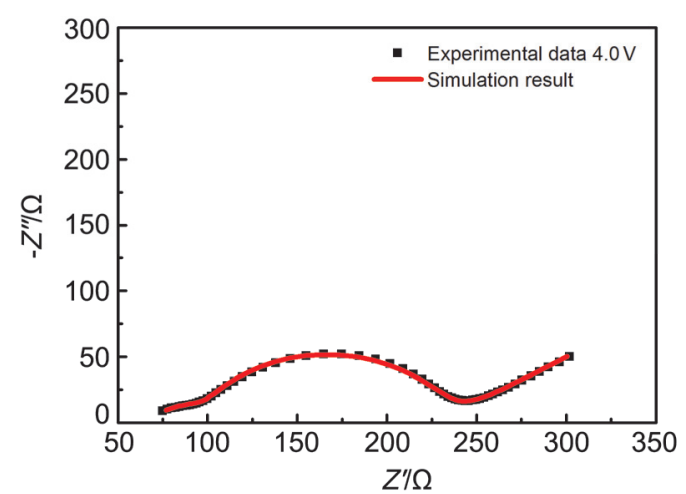

图 $114.0 \mathrm{~V}$ 下实验曲线与拟合曲线对比图

Figure 11 A comparison of the EIS data that were collected at $4.0 \mathrm{~V}$ with the simulated data

图 $12 \mathrm{a}$ 为拟合获得的 $R_{\mathrm{el}}$ 随电位变化的规律. 可以发 现，充放电过程中 $R_{\mathrm{el}}$ 在 $3.5 \sim 3.8 \mathrm{~V}$ 之间基本不变, 在电 
位 $3.8 \sim 4.1 \mathrm{~V}$ 之间持续增加，在 $4.1 \sim 4.3 \mathrm{~V}$ 基本不变; 放电过程中, $R_{\mathrm{el}}$ 在 $4.3 \sim 3.7 \mathrm{~V}$ 之间持续增加, 在 $3.7 \sim 3.5$ $\mathrm{V}$ 之间基本不变. 根据固态电解质的砖层模型 ${ }^{[39]}$, 晶粒 电导率用 $\sigma_{\mathrm{b}}$ 表示, 晶界电导率用 $\sigma_{\mathrm{gb}}$ 表示, 当 $\sigma_{\mathrm{b}}>>\sigma_{\mathrm{gb}}$ 时, 沿晶粒间界的电导可以忽略, 电流通过晶粒并穿越 晶粒间界，其行为可用复阻抗率来表示:

$$
\rho_{\mathrm{t}}=\rho_{\mathrm{b}}+\frac{1}{3} x_{\mathrm{gb}} \rho_{\mathrm{gb}}
$$

其中, $\rho_{\mathrm{t}}$ 为总阻抗, $\rho_{\mathrm{b}}$ 为晶粒阻抗, $\rho_{\mathrm{gb}}$ 为晶界阻抗, $x_{\mathrm{gb}}$ 为 晶界的体积分数. 当 $\sigma_{\mathrm{gb}}>>\sigma_{\mathrm{b}}$ 时, 电导以沿晶粒间界为 主, 复导纳为:

$$
\Psi_{\mathrm{t}}=\Psi_{\mathrm{b}}+\frac{2}{3} x_{\mathrm{gb}} \Psi_{\mathrm{gb}}
$$

其中, $\psi_{\mathrm{t}}$ 为总导纳, $\psi_{\mathrm{b}}$ 为晶粒导纳, $\psi_{\mathrm{gb}}$ 为晶界导纳. 对于 硫化物固体电解质, $R_{\mathrm{b}}>>R_{\mathrm{gb}}$, 即 $\sigma_{\mathrm{gb}}>>\sigma_{\mathrm{b}}$, 所以电解 质的复导纳符合公式(2), 这种情况下, 阻抗图中高频区 域仅有一个圆弧, 无法得到电解质的微结构信息 ${ }^{[40]}$. 本 研究中高频区域始终呈现一个半圆, 结果与其理论预测 一致. 我们推测, $R_{\mathrm{el}}$ 在高电位 $3.8 \sim 4.3 \mathrm{~V}$ 之间的持续增 加一方面是由于 LGPS 为亚稳相, 在高电位下脱出 $\mathrm{Li}^{+}$, 分解成 $\mathrm{GeS}_{2} 、 \mathrm{~S}$ 和 $\mathrm{P}_{2} \mathrm{~S}_{5}$, 导致晶粒电阻增大 ${ }^{[41]}$. 另一方 面, 电压区间 3.8 4.3 V 处于充放电平台附近, 浓差极 化较大, 晶体内部产生应力, 可能导致部分晶粒破碎, 产生更多的晶界, 导致晶界阻抗增大. 但晶界阻抗增大 的程度还不足以使阻抗谱中与其相应的半圆与晶粒阻 抗的半圆分离, 所以表现为 $R_{\mathrm{el}}$ 的增大.

图 $12 \mathrm{~b}$ 为拟合获得的 $R_{\mathrm{ct}}$ 随电位的变化. 电荷传递电 阻 $R_{\mathrm{ct}}$ 满足以下式子 ${ }^{[42]}$ :

$$
R_{\mathrm{ct}}=\frac{1}{f F A k_{\mathrm{s}} x^{0.5}(1-x)^{0.5}}
$$

在上式中, $f=\mathrm{F} / \mathrm{RT}$, 其中 $f$ 为电化学反应常数, $F$ 为法拉 第常数, $R$ 为气体常数, $T$ 为绝对温度, $k_{\mathrm{s}}$ 为非均匀度常 数, $A$ 为总的极片电活性表面积. 从式(3)可知, 在嵌锂 度 $x<0.5$ 时, 电荷传递电阻 $R_{\mathrm{ct}}$ 随着嵌锂度 $x$ 的减小不 断增大; 在嵌锂度 $x>0.5$ 时, 电荷传递电阻 $R_{\mathrm{ct}}$ 随着嵌锂 度 $x$ 的减小不断减小, 即电荷传递电阻 $R_{\mathrm{ct}}$ 在嵌锂度 $x$ $=0.5$ 时具有最小值, 对应于本文 $4.3 \mathrm{~V}$ 时, $x=0.5$ 且 $R_{\mathrm{ct}}$ 具有最小值. 从图 $12 \mathrm{~b}$ 中可以看出, 在首次脱锂过程中, $R_{\mathrm{ct}}$ 随电极电位的升高不断减小, 直至 $4.3 \mathrm{~V}$ 达到最小值, 在嵌锂过程中呈现可逆的变化过程, 此变化趋势完全符 合(3)式. 这说明公式(3)可以正确表述嵌合物脱嵌锂过 程中的电荷传递变化, 即 MFS 毫无疑问地应归属于材 料的电荷传递过程.

\section{3 结论}

在本研究中, 我们合成了 LGPS 电解质, 其室温离 子电导率为 $2.02 \times 10^{-3} \mathrm{~S} / \mathrm{cm}$, 活化能为 $29.8 \mathrm{~kJ} / \mathrm{mol}$. 制
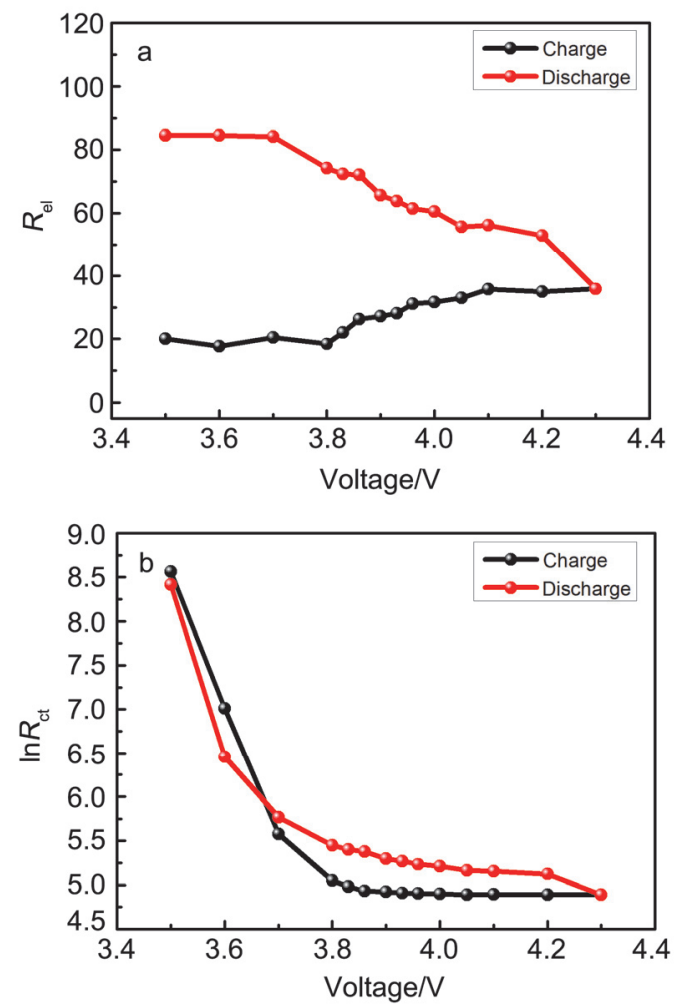

图 12 LGPS 全固态电池首次充放电过程(a) $R_{\mathrm{el}}$ 和(b) $R_{\mathrm{ct}}$ 随电极电位的 变化

Figure 12 Variation in the (a) $R_{\mathrm{el}}$ and (b) logarithm of $R_{\mathrm{ct}}$ as a function of the electrode potential during the first charging/discharging process

备了 LGPS 全固态电池, 首周放电容量可达 121.2 $\mathrm{mAh} / \mathrm{g}, 40$ 周以后库伦效率稳定在 $99.8 \%$ 左右, 经过 100 周的循环, 其放电容量有 $113.6 \mathrm{mAh} / \mathrm{g}$, 容量保持率 $93.7 \%$, 显示了较为优越的循环稳定性. 运用电化学阻 抗谱对 LGPS 全固态电池进行研究, 发现, 其典型的 EIS 谱由三个部分组成, 即高频区半圆(HFS)、中频区半 圆(MFS)和低频区斜线(LFL), HFS 归属于电解质电阻 $R_{\mathrm{el}}, \mathrm{MFS}$ 归属于电荷传递阻抗 $R_{\mathrm{ct}}, \mathrm{LFL}$ 归属于锂离子在 材料中的固态扩散过程. 通过 $R_{\mathrm{el}}$ 的拟合分析, 我们推 测, $R_{\mathrm{el}}$ 在高电位 3.8 4.3 V 之间的持续增加一方面是由 于 LGPS 在高电位下脱出 $\mathrm{Li}^{+}$, 分解成 $\mathrm{GeS}_{2} 、 \mathrm{~S}$ 和 $\mathrm{P}_{2} \mathrm{~S}_{5}$, 导致晶粒电导率下降. 另一方面可能是因为浓差极化较 大, 晶体内部产生应力, 可能导致部分晶粒破碎, 产生 更多的晶界, 晶界阻抗增大. 通过对 $R_{\mathrm{ct}}$ 的拟合, 我们发 现在首次充电过程中, $R_{\mathrm{ct}}$ 随电极电位升高不断减小, 直 至 $4.3 \mathrm{~V}$ 达到最小值, 在首次放电过程中呈现可逆的变 化过程，其变化规律符合公式(3)对 $R_{\mathrm{ct}}$ 的描述. 因此，对 于 LGPS 全固态电池而言, 提高其电化学性能的关键在 于提升 LGPS 高电位下的稳定性和降低 $R_{\mathrm{ct}}$.

\section{4 实验部分}

\section{1 材料制备}

LGPS 按文献 ${ }^{[31]}$ 中的方法合成, 首先将 $\mathrm{Li}_{2} \mathrm{~S}$ (Alad- 
din, 99.98\%)， $\mathrm{P}_{2} \mathrm{~S}_{5}$ (Aladdin, 99\%) 和 $\mathrm{GeS}_{2}$ (Aladdin, 99.999\%)按物质的量比 $5: 1: 1$ 高能球磨 $30 \mathrm{~min}$, 然后 在 $280 \mathrm{MPa}$ 的压力下压成片, 转移到管式炉中, 在 $550{ }^{\circ} \mathrm{C}$ 处理 $8 \mathrm{~h}$, 此过程均在手套箱中进行. 我们在商 业化活性材料 $\mathrm{LiNi}_{1 / 3} \mathrm{Co}_{1 / 3} \mathrm{Mn}_{1 / 3} \mathrm{O}_{2}$ (厦门铇业, 99\%)表面 包覆了一层 $\mathrm{LiNbO}_{3}$, 包覆方法是在文献 ${ }^{[43]}$ 的基础上进 行了改进. 首先, 将 $1 \mathrm{mg}$ 锂金属(成都建中, 电池级)加 入盛有 $4.6 \mathrm{~g}$ 无水乙醇(Aladdin, AR)的烧杯中, 然后加入 $44.9 \mathrm{mg}$ 乙醇铌 $(\mathrm{V})(\mathrm{Alfa}, 99.99 \%)$, 磁力搅拌 $2 \mathrm{~h}$, 然后加 入 $1 \mathrm{~g} \mathrm{LiNi}_{1 / 3} \mathrm{Co}_{1 / 3} \mathrm{Mn}_{1 / 3} \mathrm{O}_{2}$, 磁力搅拌 $6 \mathrm{~h}$, 在 $50{ }^{\circ} \mathrm{C}$ 下加 热搅拌至无水乙醇将要挥发完毕, 转移至鼓风干燥箱干 燥 6 h. 然后在管式炉中 $400{ }^{\circ} \mathrm{C}$ 处理 $30 \mathrm{~min}$, 此过程通 氧气气流. 正极的制备是将 LGPS、LNCM111 和炭黑(国 药集团化学试剂有限公司, AR)按质量比 $60: 30: 10$ 以 $450 \mathrm{r} / \mathrm{min}$ 球磨 $2 \mathrm{~h}$.

\section{2 结构和形貌表征}

XRD 测试采用德国布鲁克 (BRUKER) 公司的 $\mathrm{D} / \mathrm{Max}-3 \mathrm{~B}$ 型 $\mathrm{X}$ 射线衍射仪, LGPS 的 $2 \theta$ 角范围为 $10^{\circ} \sim 90^{\circ}$, LNCM111 的 $2 \theta$ 角范围为 $10^{\circ} \sim 70^{\circ}$. 此外, 由 于 LGPS 在空气中不稳定, 所以进行 XRD 测试时先在手 套箱中用聚酰亚胺薄膜将其密封在载玻片上. 另外, 为 了对比, 将聚酰亚胺薄膜直接贴在载玻片上进行了 XRD 测试(图 1 中 Blank). SEM 图采用日立 Hitachi S-4800 扫描电子显微镜收集, 且进行了 Mapping 测试.

\section{3 电池组装和电化学测试}

在手套箱中进行全阻塞电池 SS/LGPS/SS 和半阻塞 电池 SS/LGPS/Li 的制备. 取 $150 \mathrm{mg}$ LGPS 使其均匀分 布 $13 \mathrm{~mm}$ 在冷压模具中, 在 $360 \mathrm{MPa}$ 下进行冷压, 保压 $2 \mathrm{~min}$. 取出片后, 全阻塞电池两端放置不锈钢片, 半阻 塞电池一端放置不锈钢片, 一端放置直径 $12 \mathrm{~mm}$ 的锂片 (成都建中, 电池级), 然后分别封装在 CR2025 的电池壳 中, 密封的电池壳可以持续的为电池提供一个稳定的压 力. 全阻塞电池用于离子电导率的测试, 半阻塞电池用 于 LGPS 对锂稳定性的测试.

在手套箱中进行全固态电池的组装. 先取 $4.5 \mathrm{mg}$ 正 极材料, 使其均匀分布在直径 $13 \mathrm{~mm}$ 压片模具中, 然后 取 $100 \mathrm{mg}$ LGPS 使其均匀分布在正极材料的上方, 在 $360 \mathrm{MPa}$ 下进行冷压, 保压 $2 \mathrm{~min}$. 取出片后, 在电解质 另一面贴上直径 $12 \mathrm{~mm}$ 的锂片, 然后封装在 CR2025 的 电池壳中.

DC 测试在 LANHE 蓝电电池检测系统(武汉蓝电 科技股份有限公司)上完成, 充放电电压范围是 $2.7 \sim 4.3$ $\mathrm{V}$, 充放电倍率为 $0.05 \mathrm{C}$. CV 测试在电化学工作站 (CHI660C, 上海辰华仪器厂) 上完成, 电位扫描速度为 $0.5 \mathrm{mV} / \mathrm{s}$, 电压范围为 $2.7 \sim 4.3 \mathrm{~V}$. EIS 测试在电化学工 作站 Autolab PGSTAT302N 上完成, 测试频率范围为 $100 \mathrm{kHz} \sim 0.1 \mathrm{~Hz}$, 施加的交流信号振幅为 $5 \mathrm{mV}$, 电压 范围为 $2.7 \sim 4.3 \mathrm{~V}$.

\section{References}

[1] Goodenough, J. B.; Kim, Y. Chem. Mater. 2010, 22, 587.

[2] Jin, Z. Q.; Xie, K.; Hong, X. B. Acta Chim. Sinica 2014, 72, 11 (in Chinese). (金朝庆, 谢凯, 洪晓斌, 化学学报, 2014, 72, 11.)

[3] Liu, J.; Xu, J. Y.; Lin, Y.; Li, J.; Lai, Y. Q.; Yuan, C. F.; Zhang, J.; Zhu, K. Acta Chim. Sinica 2013, 71, 869 (in Chinese). (刘晋, 徐俊 毅, 林月, 李劼, 赖延清, 袁长福, 张锦, 朱凯, 化学学报, 2013, 71, 869.)

[4] Bachman, J. C.; Muy, S.; Grimaud, A.; Chang, H. H.; Pour, N.; Lux, S. F.; Paschos, O.; Maglia, F.; Lupart, S.; Lamp, P. Chem. Rev. 2016, $47,140$.

[5] Lin, D.; Liu, Y.; Cui, Y. Nat. Nanotechnol. 2017, 12, 194.

[6] Qiu, Z.; Zhang, Y.; Xia, S.; Dong, P. Acta Chim. Sinica 2015, 73, 992 (in Chinese). (邱振平, 张英杰, 夏书标, 董鹏, 化学学报, 2015, 73, 992.)

[7] Liu, W. Y.; Fu, Z. W.; Qin, Q. Z. Acta Chim. Sinica 2004, 62, 2223 (in Chinese). (刘文元，傅正文，秦启宗，化学学报，2004，62, 2223.)

[8] Deng, Z.; Mo, Y.; Ong, S. P. NPG Asia Mater. 2016, 8, e254.

[9] Manthiram, A.; Yu, X.; Wang, S. Nat. Rev. Mater. 2017, 2, 16103.

[10] Ramakumar, S.; Deviannapoorani, C.; Dhivya, L.; Shankar, L. S.; Murugan, R. Prog. Mater. Sci. 2017, 88, 325.

[11] Xu, H. H.; Wang, S. F.; Wilson, H.; Zhao, F.; Manthiram, A. Chem. Mater. 2017, 29, 7206.

[12] Zhang, B.; Rui, T.; Yang, L.; Zheng, J.; Zhang, K.; Mo, S.; Zhan, L.; Feng, P. Energy Storage Mater. 2018, 10, 139.

[13] He, X.; Zhu, Y.; Mo, Y. Nat. Commun. 2017, 8, 15893.

[14] Fergus, J. W. J. Power Sources 2010, 195, 4554.

[15] Minami, T.; Hayashi, A.; Tatsumisago, M. Solid State Ion. 2006, $177,2715$.

[16] Luo, W.; Gong, Y.; Zhu, Y.; Li, Y.; Yao, Y.; Zhang, Y.; Fu, K. K.; Pastel, G.; Lin, C. F.; Mo, Y. Adv. Mater. 2017, 29, 1606042.

[17] Zhu, Y.; He, X.; Mo, Y. J. Mater. Chem. A 2016, 4, 3253.

[18] Wang, Q.; Wen, Z.; Jin, J.; Guo, J.; Huang, X.; Yang, J.; Chen, C. Chem. Commun (Camb). 2016, 52, 1637.

[19] Li, Y.; Zhou, W.; Chen, X.; Lü, X.; Cui, Z.; Xin, S.; Xue, L.; Jia, Q.; Goodenough, J. B. Proc. Natl. Acad. Sci. U. S. A. 2016, 113, 13313.

[20] Kato, A.; Hayashi, A.; Tatsumisago, M. J. Power Sources 2016, 309, 27.

[21] Zhu, Y.; He, X.; Mo, Y. ACS Appl. Mater. Interfaces 2015, 7, 23685.

[22] Sharafi, A.; Kazyak, E.; Davis, A. L.; Yu, S.; Thompson, T.; Siegel, D. J.; Dasgupta, N. P.; Sakamoto, J. Chem. Mater. 2017, 29, 7961.

[23] Takada, K. Langmuir 2013, 29, 7538.

[24] Yokokawa, H. Solid State Ion. 2016, 285, 126.

[25] Long, P.; Xu, Q.; Peng, G.; Yao, X.; Xu, X. ChemElectroChem 2016, 3,764 .

[26] Wan, H.; Gang, P.; Yao, X.; Jing, Y.; Ping, C.; Xu, X. Energy Storage Mater. 2016, 4, 59.

[27] Nagao, M.; Kitaura, H.; Hayashi, A.; Tatsumisago, M. J. Power Sources 2009, 189, 672.

[28] Gao, J.; Zhao, Y. S.; Shi, S. Q.; Li, H. Chinese Phys. B 2016, 25, 35.

[29] Haruyama, J.; Sodeyama, K.; Tateyama, Y. ACS Appl. Mater. Interfaces 2017, 9, 286.

[30] Tatsumisago, M.; Nagao, M.; Hayashi, A. J. Asian Ceram. Soc. 2013, 1,17 .

[31] Noriaki, K.; Kenji, H.; Yuichiro, Y.; Masaaki, H.; Ryoji, K.; Masao, Y.; Takashi, K.; Yuki, K.; Shigenori, H.; Koji, K. Nat. Mater. 2011, 10,682 .

[32] Amani, J. A.; Koppe, T.; Hofsäss, H.; Vetter, U. Phys. Rev. Appl. 2015, 4, 044007.

[33] Zhuang, Q. C.; Xu, S. D.; Qiu, X. Y.; Cui, Y. L.; Fang, L.; Sun, S. G. Prog. Chem. 2010, 22, 1044 (in Chinese). (庄全超, 徐守冬, 邱祥 云, 崔永丽, 方亮, 孙世刚, 化学进展, 2010, 22, 1044.)

[34] Siroma, Z.; Sato, T.; Takeuchi, T.; Nagai, R.; Ota, A.; Ioroi, T. J. Power Sources 2016, 316, 215.

[35] Boulineau, S.; Courty, M.; Tarascon, J. M.; Viallet, V. Solid State Ion. 2012, 221, 1 .

[36] Han, F.; Gao, T.; Zhu, Y.; Gaskell, K. J.; Wang, C. Adv. Mater. 2015, $27,3473$.

[37] Qiu, X. Y.; Zhuan, Q. C.; Zhang, Q. Q.; Cao, R.; Qiang, Y. H.; Ying, P. Z.; Sun, S. G. J. Electroanal. Chem. 2013, 688, 392.

[38] Liu, W.; Sun, D.; Feng, T.; Xia, J.; Wang, Q.; Jiang, D. J. Ceram. 2013, 34, 555 (in Chinese). (刘薇, 孙大志, 冯涛, 夏金峰, 王琪, 蒋丹宇, 陶瓷学报, 2013, 34, 555.) 
[39] Verkerk, M.; Middelhuis, B.; Burggraaf, A. Solid State Ion. 1982, 6, 159.

[40] Näfe, H. Solid State Ion. 1984, 13, 255.

[41] Mo, Y.; Ong, S. P.; Ceder, G. Chem. Mater. 2012, 24, 15.
[42] Levi, M. D.; Gamolsky, K.; Aurbach, D.; Heider, U.; Oesten, R. Electrochim. Acta 2000, 45, 1781.

[43] Ohta, N.; Takada, K.; Sakaguchi, I.; Zhang, L. Q.; Ma, R. Z.; Fukuda, K.; Osada, M.; Sasaki, T. Electrochem. Commun. 2007, 9, 1486.

(Cheng, B.; Fan, Y.) 